\title{
Wandering Spleen
}

National Cancer Institute

\section{Source}

National Cancer Institute. Wandering Spleen. NCI Thesaurus. Code C85224.

A condition characterized by an abnormal spleen position due to loss, weakness, or

malformation of one or more of the ligaments that hold the spleen in its normal position

in the left upper abdomen. It may present as a birth defect or follow injuries or

pregnancy. Signs and symptoms include abdominal discomfort and splenomegaly. 